problem here is the shortage of observational constraints that can be related directly to convection rather than just to the rigidity of the plates. Two possibilities suggested by Richter and Parsons, however, are free-air gravity anomalies and residual elevation anomalies (differences between measured ocean floor depths and the corresponding depths to be expected as the result of the cooling of the lithosphere as it moves away from the ridge). As far as the roll-like pattern of convection is concerned, the best place to look for such evidence would probably be the Pacific where the plate velocity is relatively high and where no significant change in spreading direction has apparently occurred for $40-45$ million years. But the region in which anomalies have been most closely observed so far is the North Atlantic where the slower spreading rate makes roll-like features less likely to be observed (nor are they) and thus where the small-scale convection pattern is more likely to take the form of upwelling and downwelling spouts. This mismatch is unfortunate, but with luck only temporary.

\section{Peanuts and cancer}

\section{from Arie J. Zuckerman}

THE geographical pathology of primary cancer of the liver presents intriguing features. Liver cell cancer is rare in North America, Europe, the Soviet Union and Australia and it seems to be relatively infrequent in Central and South America. In contrast, hepatocellular carcinoma is common in many communities in Africa and South-East Asia and probably less so in Japan. In some parts of the tropics, liver cell cancer is the commonest type of cancer in adult males and it is often, though not always, found in patients with cirrhosis. Epidemiological and experimental evidence is accumulating to implicate natural hepatocarcinogens as contaminants in foodstuffs and hepatitis $B$ virus in the aetiology of primary liver cancer.

The carcinogenic potential of a number of mycotoxins and pyrrolizidine alkaloids is now well established in experimental animals. The aflatoxins form a group of closely related substances produced by certain strains of moulds of Aspergillus flavus-oryzae. These substances have been shown to have marked toxic and carcinogenic effects on the liver and other tissues of experimental animals. The cytotoxicity of aflatoxins on human embryo liver cells in cultures has also been demonstrated; aflatoxin inhibited DNA, RNA and protein synthesis in such cultures (Zuckerman et al., Br. J. exp.
Path., 49, 33; 1968). The order of toxicity of aflatoxin $B_{1}$ and $G_{1}$ in human liver cells was similar to the order of carcinogenicity in rats.

The mould Aspergillus favus readily grows on groundnuts (peanuts) and grains when they are stored traditionally in the open, in hot, humid conditions. Indeed approximate relationships have been reported between aflatoxin contamination of market food samples and the incidence of primary liver cancer in Uganda, Swaziland and Thailand. Peers and Linsell $(B r . J$. Cancer, 27,$473 ; 1973$ ) found a significant correlation in the incidence of liver cell cancer and the amount of aflatoxin actually consumed in a study carried out in three different altitude areas in the Muranga district of Kenya. In the low altitude area, below 5,250 foot above sea level, the incidence of liver cancer was greater and the aflatoxin levels in the food samples were higher. Van Rensburg and colleagues ( $S$. Afric. J. Med., 48, 250a; 1974) have also shown that aflatoxin consumption by Africans living in the Inhambane district of Mozambique was remarkably high, correlating well with the high prevalence of liver cancer in that area. Peanuts and, in some areas of Africa, maize form the staple diet of Africans and the consumption of food contaminated with fungal matabolic products is likely to be high. Yet it would be prudent to note that the quantity of aflatoxin that would need to be ingested by an individual in the form of contaminated peanuts, assuming that the dose is similar to that needed to cause experimental necrosis of the liver seen in animals, is in gross excess of that likely to be consumed, amounting to several kilograms per day (Higginson, Tumours of the Liver, Pack and Islami; Berlin, 1970).

The apparently high incidence of infection with hepatitis $\mathbf{B}$ virus in chronic liver disease and especially macronodular cirrhosis and primary liver cancer in many regions in Africa and South-East Asia also implies the possibility of some form of an aetiological association. That a strong correlation has been found in some studies cannot be denied, but there are also a number of conflicting reports from different regions. The most recent study by Bagshawe and her colleagues in Kenya (Br. J. Cancer, 31, 581; 1975) revealed that in the Muranga district (see above) no significant differences were found in the prevalence of hepatitis B surface antigen $(3.6 \%)$ between the low altitude areas, with a relatively high incidence of primary liver cancer, and the high altitude area $(2.7 \%)$ with a low incidence of the tumour.

Liver cell cancer is probably the cumulative result of many factors, and the roles of viral and parasitic infec- tion, chemical carcinogens, nutritional and other environmental factors remain to be determined.

\section{QSOs still problematical}

from R. F. Carswell

THE origin of absorption lines in some QSOs remains something of a puzzle. Perhaps the only thing that is clear is that there are absorbing clouds some distance from the QSO, which have redshifts which are usually less than that of the emitting object itself. The velocity differences are usually less than about one-tenth the speed of light, but in one well established case the velocity is about half the speed of light. Even so, the redshifts of the QSOs in which these absorptions are seen are so high that, even with these large velocities relative to the QSO, the clouds are still receding from us. Study of the absorption line regions is clearly important, since if they are intervening galaxies then they can give us some idea of the nature of galaxies during the early Universe, and if they are intrinsic to the QSOs then they must be telling us something about the properties of the QSOs themselves. There are a number of problems in interpreting the observational data however, which leave the situation unclear.

The first major problem is that of identifying the redshifts of the absorption clouds, given a spectrum which may contain anything up to 200-300 absorption lines. A single QSO may have many absorbing clouds, at many very different redshifts, each with differing excitation properties so that it is not always possible to say definitely what lines, or redshift systems, must be present. What is usually done in the case of a newly discovered absorption line QSO is to try all reasonable candidate lines at all possible redshifts, and see if any physically consistent, and statistically significant, absorption systems are present. Often this is done on a fairly subjective basis, but a computer program developed by Aaronson, McKee and Weisheit (Astrophys. J., 198, 13; 1975) may help bring a consistent approach to the subject. The authors have analysed all previously published data on absorption line QSOs looking for candidate absorption systems using a coherent set of atomic physics and ionisation equilibrium criteria, and checked the probability of finding such a system by chance. They show that the number of well-established systems is in fact much smaller than had previously been believed, notably in very absorp- 